\title{
ORIGINALBEITRAG \\ Effektivverzinsung und Volatilität bei Finanzierung mit Zinsbindung und variablen Zinsen
}

Eine empirische Untersuchung für Deutschland

\author{
Jörg Clostermann · Franz Seitz $(\mathbb{D}$
}

Eingegangen: 13. November 2018 / Überarbeitet: 13. September 2019 / Angenommen: 24. September 2019 / Online publiziert: 17. Oktober 2019

(C) Der/die Autor(en) 2019

Zusammenfassung Wir stellen einer festen Zinsbindung von 10 Jahren bei Wohnungsbaukrediten eine monatliche an einem Kurzfristzins angepasste Zinsbelastung bei unterschiedlichen Rückzahlungsfristen gegenüber. Dafür werten wir Monatsdaten seit den 1950er Jahren für Deutschland aus. Damit greifen wir mehrere Zinszyklen, Zinserhöhungs- und Zinssenkungsphasen ab. Im Ergebnis finden wir, dass in der überwiegenden Mehrzahl der Fälle kurzfristig variable Zinsen eine geringere Zinsbelastung nach sich ziehen. Und diese weisen auch noch geringere Schwankungen auf.

Schlüsselwörter Festzinsbindung · Zinsspread · Variable Zinsen · Zinsstruktur · Immobilienfinanzierung

JEL E43 $\cdot$ E47 $\cdot$ G21

Das Papier basiert auf einem Projekt, das die beiden Autoren mit der Neuwirth Finance GmbH durchgeführt haben. Wir danken den Mitarbeitern und speziell Kurt Neuwirth für die gewährte Unterstützung und wertvolle Hinweise sowie die fruchtbare Zusammenarbeit. Des Weiteren danken wir den Teilnehmern des Frühjahrs-Workshops 2019 des ROME-Netzwerks, A. Koch, S. Sebastian, G. Ziebarth und 2 anonymen Gutachtern für hilfreiche Anregungen und Kommentare.

J. Clostermann

Technische Hochschule Ingolstadt, Esplanade 10, 85049 Ingolstadt, Deutschland

E-Mail: Joerg.Clostermann@thi.de

F. Seitz $(\bowtie)$

Ostbayerische Technische Hochschule Weiden, Hetzenrichter Weg 15, 92637 Weiden, Deutschland

E-Mail: f.seitz@oth-aw.de 


\title{
Effective interest rates and volatility for fixed-rate and adjustable-rate mortages
}

An empirical investigation for Germany

\begin{abstract}
We compare fixed-rate (10 years) and adjustable-rate loans (mortgages) with different repayment periods for Germany. The sample starts in the 1950s, i.e. we include several interest rate cycles, periods with increasing and periods with decreasing interest rates, and use monthly data. Our findings reveal that in the overwhelming majority of cases variable rates yield lower interest rate costs. Furthermore, the total volatility of these costs is lower than that of fixed-rate loans.
\end{abstract}

Keywords Fixed-rate mortgage $\cdot$ Adjustable-rate mortage $\cdot$ Term structure of interest rate $\cdot$ Interest rates $\cdot$ Risk

\section{Einleitung, Problemstellung}

„Zwar wird Ihnen bei kurzen Zinsbindungsfristen gewöhnlich ein etwas geringerer Darlehenszinssatz eingeräumt, bei Ablauf der Zinsbindungsfrist drohen jedoch möglicherweise erheblich höhere Zinsbelastungen, die das zur Verfügung stehende Haushaltsbudget leicht übersteigen können. Daher sollten Sie (...) möglichst lange Zinsbindungen (...) wählen.“1 „Hohes Zinserhöhungsrisiko durch zu kurze Zinsbindung.“2 „Nutzen Sie die aktuellen Minizinsen und schreiben Sie die Konditionen langfristig fest". ${ }^{3}$ Diese Zitate stehen stellvertretend für Formulierungen und Empfehlungen von Immobilienfinanzierern und Banken, die die Vorteile einer festen Zinsbindung (fixed-rate mortgages, FRM) - in Deutschland in der Regel zehn Jahre - gegenüber kurzfristig flexiblen Zinskonditionen (adjustable-rate mortgages, ARM) anpreisen.

In diesem Zusammenhang gilt es zu beachten, dass langfristige Hypothekarkredite mit ca. 50\% den größten Anteil der Kredite der Banken in Deutschland an inländische Unternehmen und Privatpersonen darstellen und für private Haushalte die Kredite für den Wohnungsbau die mit Abstand bedeutendste Verschuldungsform sind. ${ }^{4}$ In Deutschland ist der klassische Immobilienkredit ein Annuitätendarlehen mit einer Laufzeit von mindestens 5 Jahren und Zinsbindung. Stand März 2019 beliefen sich die gesamten Kredite für den Wohnungsbau an Privatpersonen und Unternehmen auf 1,4 Bio. $€$. Davon hatten $97 \%$ der Kredite eine Laufzeit von mindestens 5 Jahren (Deutsche Bundesbank 2019a, S. 32*; Deutsche Bank Research 2019, S. 2). Von den gesamten neu vergebenen Wohnungsbaukrediten (=Neugeschäfte) an private Haushalte entfallen in Deutschland fast $80 \%$ auf ursprüngliche Zinsbindungen von über fünf Jahren und etwa $45 \%$ auf solche von über 10 Jahren (Stand April

\footnotetext{
1 Siehe https://www.aktuelle-bauzinsen.info/zinsbindung-baufinanzierung.html.

2 https://www.test.de/Baufinanzierung-Elf-teure-Fehler-und-wie-Sie-diese-vermeiden-5227717-0/.

3 http://www.geldsparen.de/baufinanzierung/baukredit-feste-oder-variable-zinsen.php.

4 Diese Beobachtungen gelten im Prinzip weltweit.
} 


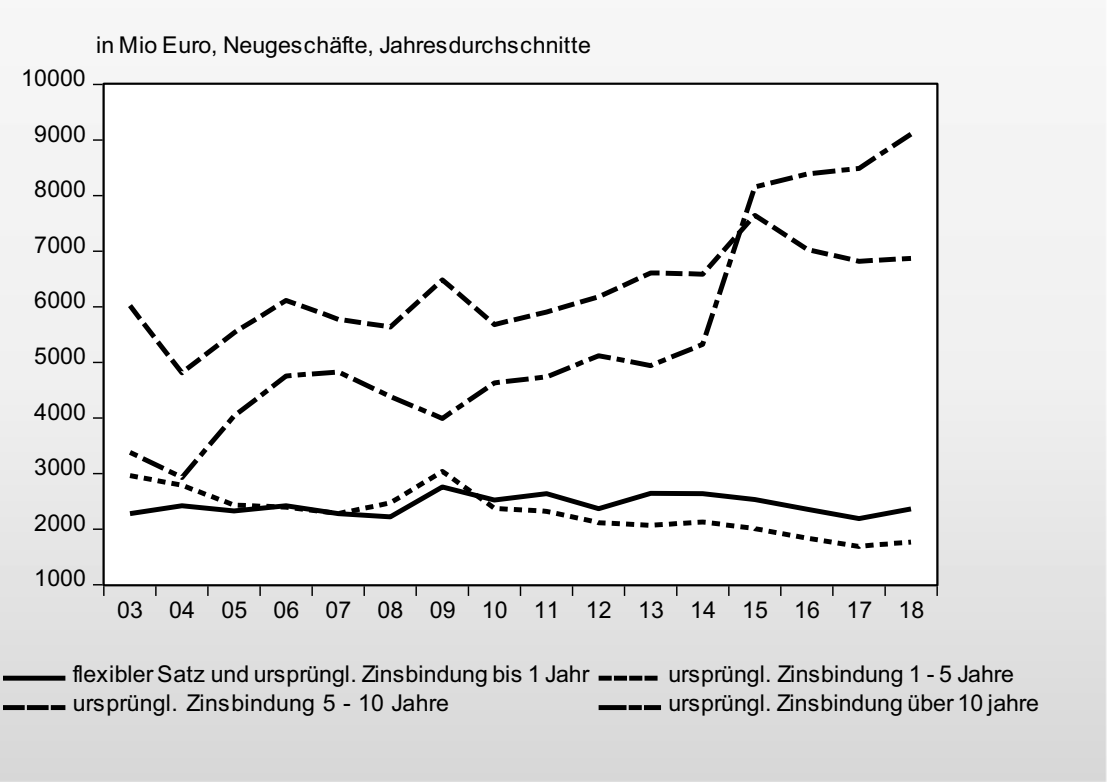

Abb. 1 Wohnungsbaukredite an private Haushalte in Deutschland nach Zinsbindung. Quelle: Deutsche Bundesbank

2019, siehe Deutsche Bundesbank 2019b, S. 4). ${ }^{5}$ Die Situation in Deutschland bei den Neugeschäft-Volumina zeigt einen ansteigenden Trend bei den längeren Zinsbindungen und eher rückläufige Tendenzen bei den kürzeren Zinsbindungen bzw. variablen Anteilen (siehe Abb. 1). Die Effektivzinsen von Wohnungsbaukrediten fallen seit der Finanzkrise 2008 auf breiter Front. Bis Ende 2014 wiesen dabei die längeren Zinsbindungen die höchsten Sätze auf. Ab 2015 drehte sich das um, sodass seither auf niedrigem Niveau die höchsten Zinsen bei den kurzfristigen Bindungen und variablen Kontrakten vorherrschen (siehe Abb. 2).

Auf einschlägigen Internetportalen in Deutschland, die Kreditvergleiche von Immobilienkrediten anbieten, werden nahezu ausschließlich Annuitätenkredite mit Zinsbindungen angeboten, was die Vorliebe der Deutschen für berechenbare Rückzahlungsraten unterstreicht. Dies steht im Kontrast zur Schweiz. Hier findet man von Finanzdienstleistern und Banken Immobilienkreditangebote, die zwar durchaus auch Laufzeiten von 5 Jahren und mehr aufweisen können, der Kunde allerdings die Option hat, eine Zinsbindung zu wählen oder sich für einen variablen Zins zu entscheiden, der mit einem Geldmarktzins synchronisiert ist (SNB 2019). Die Kreditform mit variablem Zins hat sich in der Schweiz seit der Jahrtausendwende etabliert.

\footnotetext{
5 In Australien, Irland, Großbritannien und südeuropäischen Ländern dagegen dominieren Hypothekarkredite mit anpassungsfähigen Zinsen, während in Belgien, den Niederlanden, Dänemark und Schweden der Anteil „fix“ zu ,variabel“ im Zeitablauf starken Schwankungen unterliegt (Badarinza et al. 2016, Kap. 6). Der ARM-Anteil weist dabei üblicherweise gewisse Persistenzen auf.
} 


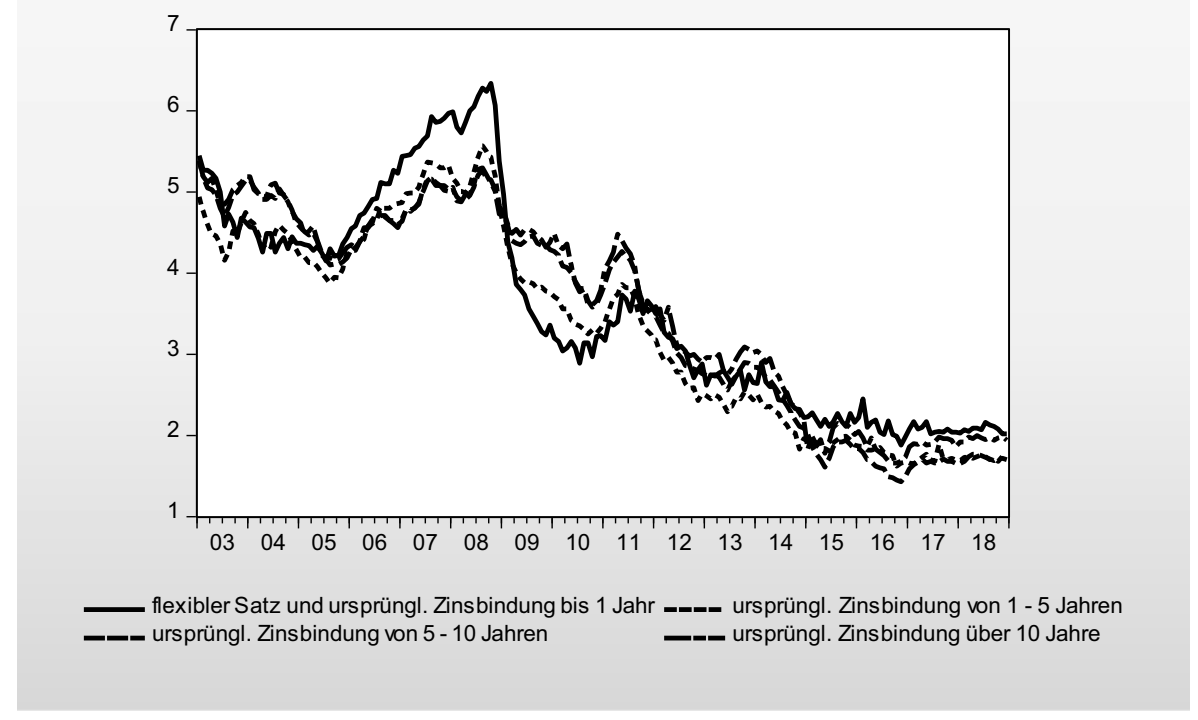

Abb. 2 Wohnungsbaukredite (Neugeschäfte) an private Haushalte in Deutschland: Effektivzinsen bei unterschiedlichen Zinsbindungen in \%. Quelle: Deutsche Bundesbank

Vor diesem Hintergrund stellt sich die Frage, wie viel die Präferenz der Deutschen für Immobilienkredite mit längeren Laufzeiten und Zinsbindung kostet? Das vorliegende Papier untersucht somit für Deutschland aus Sicht von potenziellen Kreditnehmern die Frage, ob man in der Vergangenheit besser mit einer fixen Zinsbindung im Vergleich zu variablen Zinskonditionen gefahren wäre. Dafür werden Daten zu Kurz- und Langfristzinsen ab Mitte der 1950er Jahre (auf Monatsbasis) ausgewertet und unterschiedliche Rückzahlungsperioden berücksichtigt. ${ }^{6}$ Neben den Zinskosten werden auch die Volatilität der Zinsbelastung und das Liquiditätsrisiko bzw. Zinsänderungsrisiko des Immobilienkredits mit variabler Verzinsung analysiert. Wir unterstellen dabei stets identische Wirtschaftssubjekte, die speziell auch gleiche Laufzeitpräferenzen haben. Der einzige Unterschied liegt darin, dass der eine eine feste Zinsbindung, der andere einen variablen Zins wählt. Mögliche unterschiedliche Gebühren der Finanzierungsformen werden nicht berücksichtigt. ${ }^{7}$

Der Beitrag ist folgender Maßen gegliedert. Kap. 2 gibt einen Literaturüberblick zur Thematik. Daran anschließend beschreibt Kap. 3 die verwendeten Daten. In Abschn. 4 werden die Methodik der Berechnungen und die Ergebnisse dargestellt und interpretiert. Abschn. 5 fasst zusammen, zieht einige Schlussfolgerungen und gibt einen kurzen Ausblick.

\footnotetext{
6 Zur Unterscheidung zwischen Zinsbindung und Rückzahlungsdauer siehe auch Basten et al. (2017), Kap. 2.1.

7 Dies entspricht der Situation in der Schweiz.
} 


\section{Literaturüberblick}

Einen Überblick über Wohnungsmarktstrukturen in OECD Ländern geben Andrews et al. (2011) sowie Andrews und Sánchez (2011). Badarinza et al. (2017) stellen zunächst fest, dass die Wahl einer festen Zinsbindung gegenüber einer variablen Verzinsung bei Immobilienfinanzierungen international sehr unterschiedlich ist. ${ }^{8} \mathrm{Un}$ einigkeit herrscht allerdings in der Literatur darüber, wie die Kosten von ARMs im Vergleich zu FRMs (ex-ante) tatsächlich bestimmt werden (Badarinza et al. 2016, S. 23). Dabei wird zwischen einer vergangenheitsorientierten Regel, nach der die durchschnittliche Belastung bei ARMs festgelegt wird, dem aktuellen ARM-Satz (bei liquiditätsbeschränkten Haushalten) und einer kurzfristig vorausschauenden Bestimmung des ARM-Satzes (höchstens ein Jahr) differenziert. ${ }^{9}$ Die entsprechenden Konditionen werden dann dem vorherrschenden FRM-Satz gegenübergestellt. Badarinza et al. (2017) finden eine sowohl länder- als auch zeitabhängige Entscheidung. In allen untersuchten Ländern spielt für die privaten Haushalte der aktuelle Zins eine entscheidende Rolle (siehe auch Basten et al. 2017; EZB 2007, für das Euro-Währungsgebiet). Jedoch nimmt die Bedeutung der gesamten Kosten über die Laufzeit des Darlehens (vorausschauendes Verhalten) im Zeitverlauf zu. Die Nachfrage nach längeren Zinsbindungsfristen nimmt dabei mit einem geringeren Spread zwischen lang- und kurzfristigen Zinsen zu. ${ }^{10}$

Die Mehrzahl der wissenschaftlichen Untersuchungen bezieht sich auf die USA. Badarinza et al. (2016, Kap. 6) weisen in ihrer Mehrländerstudie allerdings ausdrücklich darauf hin, dass aufgrund unterschiedlicher institutioneller Gegebenheiten die Ergebnisse für ein Land nur schwerlich auf ein anderes zu übertragen sind. Der Anteil von ARM zu FRM variiert mit dem regulatorischen Umfeld, der in der Vergangenheit vorherrschenden Schwankungen der Inflation und den Regelungen zur Hypothekenfinanzierung auf der Anbieterseite. Ehrmann und Ziegelmeyer (2017) ergänzen diese Determinanten noch um das makroökonomische Umfeld in Form der konjunkturellen Lage, des Zinsspreads und der Arbeitslosigkeit. Speziell im EuroWährungsgebiet nehmen ARMs wegen Zinssenkungserwartungen in Boomphasen, bei einem hohen Zinsspread infolge des Zinsvorteils und geringen Schwankungen der Arbeitslosenquote aufgrund eines verringerten Arbeitslosigkeitsrisikos zu.

Etliche Papiere verweisen darauf, dass persönliche Charakteristika des potenziellen Schuldners die Wahl zwischen ARMs und FRMs beeinflussen. Dabei gilt es zwischen Risiko und Kosten abzuwägen. Generell steigt der ARM-Anteil mit steigendem Einkommen (z. B. Ehrmann und Ziegelmeyer 2017). Rampini und Viswanathan (2016) finden jedoch heraus, dass auch ärmere Haushalte ARMs bzw. kürzere Zinsbindungsfristen unter Umständen präferieren (siehe auch Campbell und

\footnotetext{
${ }^{8}$ Mugerman et al. (2016) zeigen, dass bei dieser komplexen Entscheidung verhaltenswissenschaftliche Aspekte, vor allem Verfügbarkeits- und Repräsentativitätsheuristiken eine wichtige Rolle spielen. Dadurch gewinnen Änderungen des Kurzfristzinses an Bedeutung.

9 Vorstellbar wäre auch, aus der Zinsstruktur oder Terminzinsen Prognosewerte abzuleiten.

10 Siehe dazu auch EZB (2007) und Ehrmann und Ziegelmeyer (2017) für das Euro-Währungsgebiet sowie Koijen et al. (2009), die allerdings darauf verweisen, dass in den USA durch die bessere Abbildung von Risikoprämien der Bondspread ein validerer Indikator als der Laufzeitenspread ist.
} 
Cocco 2015), obwohl aus Risikogesichtspunkten eine längere Zinsbindungsfrist vorteilhaft wäre. Für derartige Haushalte tritt wegen Liquiditätsbeschränkungen das Risikomanagementmotiv gegenüber dem Finanzierungsmotiv in den Hintergrund, d. h. der Transfer von Ressourcen von der Zukunft in die Gegenwart ist für sie wichtiger als eine Glättung der Zahlungsströme über unterschiedliche Umweltzustände hinweg. Diese Schlussfolgerungen stehen im Einklang mit der Tatsache, dass die größten Kreditausfälle nach dem Platzen der Immobilienpreisblase in den USA, Irland und Spanien bei Krediten mit variabler Verzinsung kumuliert waren. Campbell und Cocco (2003) betonen die wichtige Rolle der allgemeinen Preisentwicklung, da bei Inflationsunsicherheit Kontrakte mit fester Zinsbindung einen schwankenden Realwert besitzen, während kurzfristig anpassungsfähige Zinskonditionen zu einem fixen Realwert führen, allerdings verbunden mit kurzfristig variablen Zahlungsverpflichtungen. In einem theoretischen Modell zeigen sie anhand von Simulationen, dass ARMs generell vorzuziehen sind. Dies gilt aber nur eingeschränkt für risikoaverse Haushalte mit großen Hypothekenvolumina, einem riskanten Einkommen, hohen Insolvenzkosten und/oder hoher Immobilität, da diese überdurchschnittlich hohe Risikoprämien zu tragen haben. ${ }^{11}$ Paiella und Pozollo (2007) arbeiten allerdings heraus, dass risikobezogene Haushaltscharakteristika die Entscheidung zwischen unterschiedlichen Zinsbindungen nicht erklären können. Diese wird vielmehr vom relativen Preis der Hypotheken und eventuellen Liquiditätsbeschränkungen bestimmt. Liquiditätsbeschränkte Haushalte präferieren flexible Zinskonditionen, wenn dadurch die anfänglichen Zahlungen niedrig sind. Tendenziell übersehen diese Haushalte die Gesamtkosten der Hypothek und unterschätzen das Risiko steigender Zinsen. Auf der anderen Seite überschätzen die Kreditgeber dieses Risiko, sodass die Hypothekenschuldner letztlich einen hohen Preis für die anfänglich niedrigen vertraglichen Leistungen zahlen. Damit widersprechen die Ergebnisse von Paiella und Pozollo (2007) der Effizienzmarkthypothese.

In einem ähnlichen Kontext präsentieren Basten et al. (2017) ein Modell für die Schweiz, welches die Kreditnachfrage der Haushalte und das Kreditangebotsverhalten der Banken modelliert. Sie betonen, dass die gewählte Zinsbindung von Banken und Haushalten zusammen festgelegt werden (ähnlich Foà et al. 2015; EZB 2007, S. 45). Die Haushalte sind dabei offensichtlich und in Einklang mit Badarinza et al. (2017) sowie EZB (2007) vor allem an der Minimierung der aktuellen Hypothekenkosten interessiert (ebenfalls IWF 2004, Box 2.2). Die Banken dagegen wägen ihr eigenes Zinsrisiko gegenüber der Schuldentragfähigkeit des Schuldners und dem Kreditrisiko ab. Dabei berücksichtigen sie das institutionelle Umfeld (z. B. gesetzliche Beschränkungen von Ausleihungen und Finanzierungen, insolvenzrechtliche und Rechnungslegungsvorschriften) und Marktcharakteristika (z. B. Entwicklung der „Covered Bond-Märkte“, Mortgage Backed Securities etc.) (IWF 2004, Box 2.2).

\footnotetext{
11 Campbell und Cocco (2015) präsentieren ein Modell mit Hypothekeninsolvenzen, die von diesen persönlichen Charakteristika abhängen. Ihr Modell erklärt die höheren Ausfall- bzw. Insolvenzquoten bei ARMs mit der Attraktivität derartiger Verträge für Haushalte mit höherem Arbeitseinkommensrisiko, speziell in Phasen steigender Zinsen und Preise. Die jeweilige Insolvenzwahrscheinlichkeit hängt vom ursprünglichen Zinsniveau ab: Bei ARMs (FRMs) ist sie am höchsten bei ursprünglich niedrigen (hohen) Zinsen (siehe auch IWF 2004, Box 2.2).
} 
Die makroökonomischen Folgen von ARMs relativ zu FRMs werden üblicherweise vor dem Hintergrund der stärkeren Durchschlagskraft der Zinspolitik der Zentralbanken bei ARMs diskutiert (Badarinza et al. 2016, S. 23; Calza et al. 2012; Ehrmann und Ziegelmeyer 2017, für das Euro-Währungsgebiet). ${ }^{12}$ Rubio (2011) untersucht die Rolle der Zinsbindung im Rahmen eins neukeynesianischen Dynamic Stochastic General Equilibrium (DSGE)-Modells. In diesem Modellrahmen treffen Zins(erhöhungs)schocks vor allem Kreditnehmer mit ARMs (steigende reale Zinszahlungen, sinkende Kreditsicherheiten). Lange Zinsbindungsfristen erhöhen gegeben die geldpolitische Ausrichtung - tendenziell die Gesamtwohlfahrt in dieser Ökonomie. Aber auch die Rückwirkungen auf Banken sind zu beachten. So ist für Banken mit einem hohen Anteil von FRMs davon auszugehen, dass bei künftigen (vor allem abrupten) Zinserhöhungen der Europäischen Zentralbank (ausgehend von der aktuellen Nullzinspolitik gekoppelt mit Quantitative Easing) die Zinsmargen sinken, da die kurzfristigen Einlagezinsen relativ schnell ansteigen werden, die Kreditzinsen dagegen nur in Form des Neugeschäfts. Da die größten Kreditrisiken im Rahmen der letzten Immobilienpreiskrise in den USA, Irland und Spanien bei Krediten mit variabler Verzinsung entstanden, könnten die Banken dann gegebenenfalls einem größeren Kreditausfallrisiko gegenüber stehen. Allerdings konstatieren Lerbs und Voigtländer (2018), dass durch die langen Zinsbindungen in Deutschland die Kreditausfälle aufgrund eines Zinsschocks reduziert werden, weshalb sie derzeit keine Notwendigkeit des Einsatzes makroprudenzieller Instrumente sehen.

Insgesamt kann festgehalten werden, dass über die Vorteilhaftigkeit von FRMs im Vergleich zu ARMs keine generellen Aussagen getroffen werden können. Sie hängt von Charakteristika der Kreditnehmer, dem Preissetzungsverhalten der Banken, institutionellen Gegebenheiten und makroökonomischen Bedingungen ab.

\section{Verwendete Daten}

Ziel dieser Arbeit ist es, die Finanzierungskosten für Immobilienkredite mit festen und variablen Zinsen zu vergleichen. Allerdings werden in Deutschland kaum Kredite längerer Laufzeit mit variablen Zinsen vergeben. Deshalb existieren dazu auch keine Daten. Aus diesem Grund behelfen wir uns mit einem Kunstgriff: In der Schweiz, welches ein ähnlich strukturiertes Bankensystem wie Deutschland hat, existieren solche Produkte und die Zinszeitreihen dazu. Eigene ökonometrische Berechnungen für den Fall der Schweiz zeigen, dass bei Immobilienkrediten mit einem variablen Zins dieser sich mit einem Aufschlag von ca. 120 Basispunkten parallel zu den korrespondieren Geldmarktzinssätzen bewegt. Bei der Festlegung des Zinssatzes für Immobilienkredite mit festem Zins orientieren sich die Banken dagegen offensichtlich sehr stark am Kapitalmarktzins, speziell der Rendite von Bundesanleihen. Der Aufschlag liegt hier bei ca. 145 Basispunkten, d. h. der Risikoaufschlag der Schweizer Banken bei Immobilienkrediten mit festen Zinsen ist leicht höher (statis-

\footnotetext{
12 Moench et al. (2010) untersuchen in diesem Zusammenhang, wie sich das Aufkaufprogramm der Fed in Form von MBS im Zuge der Finanzkrise auf die Wahl von ARMs im Vergleich zu FRMs ausgewirkt hat. Sie finden, dass dadurch FRMs deutlich attraktiver wurden.
} 


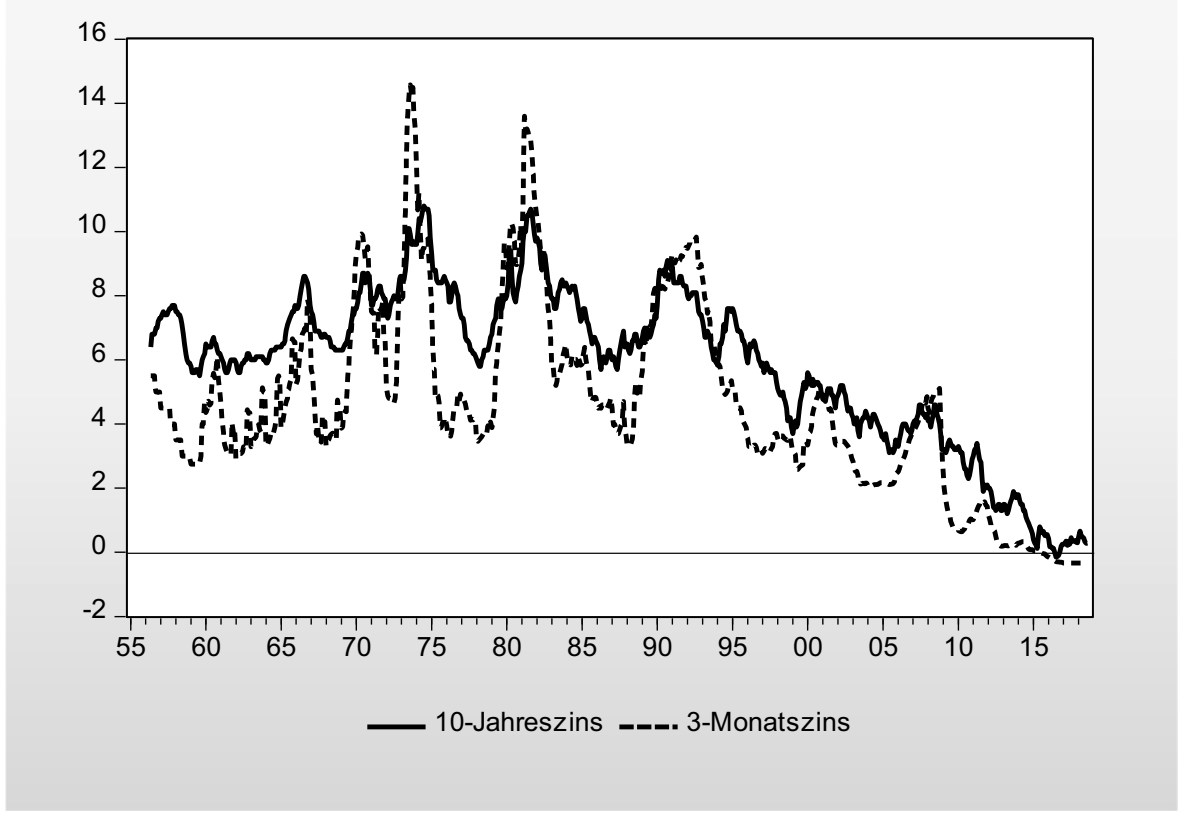

Abb. 3 Staatsanleiherendite und 3-Monats-Geldmarktsatz in \%. Quelle: Deutsche Bundesbank

tisch signifikant) als bei Immobilienkrediten mit variablen Zinsen. Diese Ergebnisse lassen die Schlussfolgerung zu, dass als Referenzzinssatz für Immobilienkredite mit variablen Zinsen ein Geldmarktzins und für Immobilienkredite mit Zinsbindung ein langfristiger Staatsanleihenzins herangezogen werden kann.

In der folgenden empirischen Analyse gehen Monatsdaten der Deutschen Bundesbank von Mai 1956 bis August 2018 ein. Damit umfasst der Datensatz 748 Beobachtungen. Als repräsentativen Zins für ein Immobiliendarlehen mit Zinsbindung und einer Laufzeit von 10 Jahren $(F R M)$ verwenden wir die Rendite 10-jähriger Bundesanleihen, da sich die deutschen Banken wohl ähnlich wie die Schweizer Banken an den Zinsen für langfristige Staatsanleihen orientieren. Diese Zeitreihe steht für Deutschland erst ab April 1973 zur Verfügung. Deshalb greifen wir für den Zeitraum von Mai 1956 bis März 1973 auf die Durchschnittsrendite aller öffentlichen Anleihen zurück. Bei den Zinskonditionen für Hypothekenkredite mit variablem Zins orientieren sich die Banken am Geldmarktzins. Aufgrund der oben geschilderten Beobachtungen und Schätzergebnisse für die Schweiz wählen wir als Referenzzinssatz für eine Finanzierung mit variabler Verzinsung $(A R M)$ von Dezember 1959 bis Dezember 1998 den 3-Monats-Geldmarktsatz unter Banken, ab Januar 1999 den 3-Monats-Euribor. Für den Zeitraum Mai 1956 bis November 1959 


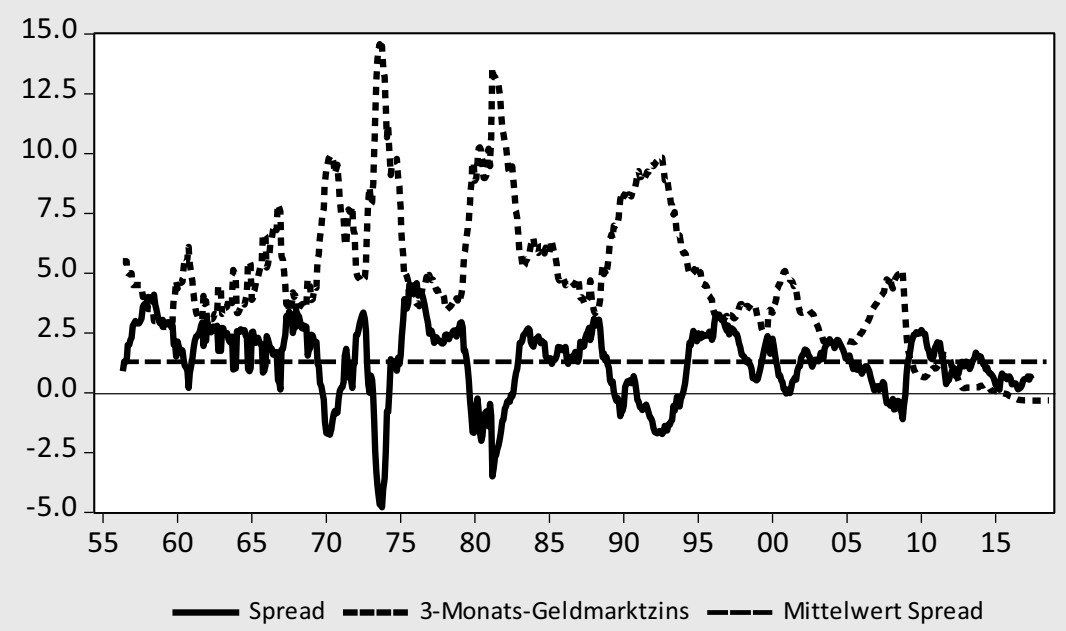

Abb. 4 Zinsspread und 3-Monats-Geldmarktzins. Anmerkung: Spread berechnet als Differenz der Rendite von Staatsanleihen und des 3-Monats-Geldmarktzinses, Prozentpunkte; Geldmarktzins in \%. Quelle: Deutsche Bundesbank

nehmen wir den Diskontsatz der Deutschen Bundesbank, da für diesen Zeitraum der 3-Monats-Geldmarktsatz nicht erhoben wurde. ${ }^{13}$

In Abb. 3 sind diese beiden Referenzzinssätze gegenübergestellt. Man erkennt die drei deutschen Zinszyklen, in denen beide Zinssätze bedingt durch die hohen Inflationsraten und der restriktiven Geldpolitik der Deutschen Bundesbank jeweils historische Höchstwerte erzielten (Erster Ölpreisschock Anfang der 70er Jahre, Zweiter Ölpreisschock Anfang der 80er Jahre, Vereinigungsboom Anfang der 90er Jahre). Seite Anfang der 90er Jahre bewegen sich beide Zinssätze im Zuge des Disinflationsprozesses abwärts. ${ }^{14}$ Darüber hinaus erkennt man, dass die Volatilität des Geldmarktzinssatzes höher ist als die Volatilität der Rendite für langfristige Staatsanleihen. Am aktuellen Rand ist der Geldmarktzins bedingt durch die Überschussliquidiät der Banken und den negativen Einlagezins der EZB negativ.

Abb. 4 zeigt die Differenz zwischen der Staatsanleiherendite und dem 3-MonatsGeldmarktsatz, den sogenannten Zinsspread. Der Verlauf des Spreads ist nahezu spiegelverkehrt zum 3-Monats-Geldmarktsatz. Insgesamt schlägt hier sozusagen die hohe Volatilität des 3-Monats-Geldmarktsatzes durch. Vor allem fällt auf: Wenn die kurzfristigen Zinsen hoch und in der Nähe ihres oberen Wendepunktes sind, ist der Spread negativ, d.h. die Zinsstrukturkurve ist negativ geneigt bzw. invers.

13 Eine Untersuchung darüber, wie deutsche Banken ihre Kreditzinsen, unter anderem bei Hypothekarkrediten, bei gegebenen (kurz- und langfristigen) Marktzinsen gestalten, findet sich in Deutsche Bundesbank (2002).

14 Das gilt auch für die entsprechenden Realzinsen und nicht nur in Deutschland, siehe z. B. Rachel und Smith (2017). 
Dies dokumentiert die starke Zinssenkungserwartung der Marktakteure. Der Mittelwert des Spreads über den gesamten Zeitraum von 60 Jahren ist positiv und liegt bei rund 1,3\%-punkten. Demnach liegt die Staatsanleiherendite im Betrachtungszeitraum durchschnittlich um 1,3\%-punkte über dem 3-Monats-Geldmarktsatz. In Deutschland lag im Durchschnitt also eine positive Fristigkeitsstruktur der Zinsen vor.

\section{Empirische Untersuchung für Deutschland}

Bei der ARM-Finanzierung kommen i.d.R. zwei Tilgungsformen zur Anwendung, das Fälligkeitsdarlehen (=endfällige Tilgung) und das Tilgungsdarlehen. ${ }^{15}$ Beim Fälligkeitsdarlehen zahlt der Kreditnehmer monatlich nur die Zinsen. Am Ende der Darlehenslaufzeit wird die vollständige Kreditsumme einmalig fällig. Beim Tilgungsdarlehen wird eine konstante monatliche Tilgungsrate festgelegt. Der Rückzahlungsbetrag des Schuldners an den Gläubiger pro Periode umfasst diese Tilgungsrate und die Zinsrate.

Bei der FRM-Finanzierung ist bei der Immobilienfinanzierung die gebräuchlichste Kreditform das Annuitätendarlehen. Die monatlichen Raten, bestehend aus Zinsen und Tilgung, sind hier konstant, wobei der Tilgungsanteil im Zeitablauf steigt, der Zinsanteil dagegen sinkt. Ein Annuitätendarlehen ist bei variabler Verzinsung nicht möglich, da die Zinsen sich kontinuierlich ändern und damit die Höhe des Rückzahlungsbetrags ex ante nicht bestimmbar ist.

Wir stellen bei dem Zinsvergleich von Krediten mit festem und variablem Zinssatz im Folgenden nur auf Fälligkeitsdarlehen ab. Clostermann und Seitz (2018) zeigen, dass Fälligkeits- und Tilgungsdarlehen in dieser Hinsicht nur marginale Unterschiede aufweisen. ${ }^{16}$

Bei den Berechnungen unterscheiden wir drei verschiedene Rückzahlungszeiträume: 10 Jahre, 20 Jahre und 30 Jahre. Während bei der Finanzierung mit variablen Zinsen der Zins sich in fixen Zeitintervallen verändert (in unserem Fall monatlich), ${ }^{17}$ unterstellen wir bei der Finanzierung mit festen Zinsen eine maximale Zinsbindung von 10 Jahren. Wird ein Objekt z. B. über 30 Jahre finanziert, muss der Kreditnehmer bei fester Zinsbindung insgesamt dreimal einen Kredit über jeweils 10 Jahre aufnehmen, was in Deutschland nicht unüblich ist. Bei Immobilienkrediten von privaten Haushalten dauert der gesamte Tilgungsprozess in der Mehrzahl der Fälle zwischen 20 und 30 Jahren. ${ }^{18}$ Grafisch explizit dargestellt wird im Folgenden nur der Fall

\footnotetext{
15 https://www.immobilienscout24.de/baufinanzierung/lexikon/darlehen-variable.html.

16 Bei der Berechnung der Effektivverzinsung gehen beim Fälligkeitsdarlehen alle Zinsen während der Laufzeit mit dem gleichen Gewicht ein, während beim Tilgungsdarlehen das Gewicht der Zinsen zum Ende der Laufzeit hin geringer wird.

17 Die Vorgehensweise mit monatlicher Zinsanpassung ist nur der Vereinfachung geschuldet. Es würden sich keine anderen Schlussfolgerungen ergeben, wenn wir eine quartalsweise Anpassung berücksichtigen würden, wie sie üblicherweise in der Praxis stattfindet.

18 Bei Immobilienkrediten von privaten Haushalten wird die anfängliche jährliche Tilgungsrate üblicherweise auf 2-2,5\% festgelegt. Bei einem Zinsniveau von $2 \%$ impliziert dies eine Tilgungsdauer von rund 35 Jahren.
} 
einer Gesamtrückzahlungsdauer von 30 Jahren; für die anderen beiden Fälle, 10 und 20 Jahre, bei denen sich ähnliche Schlussfolgerungen ergeben, siehe Clostermann und Seitz (2018).

Bei der Beurteilung, welche Finanzierungsform aus Sicht des Schuldners günstiger ist, wurde für jede Finanzierungsalternative die ex-post-Effektivverzinsung berechnet (ohne Gebühren und Ausgabeauf- oder -abschläge). Dies entspricht der tatsächlich realisierten Durchschnittsverzinsung der Kredite über die gesamte Laufzeit. Beim Fälligkeitsdarlehen entspricht die Effektivverzinsung dem arithmetischen Mittelwert der entsprechenden Referenzzinsätze. Würde ein Kreditnehmer im Januar 1960 ein Fälligkeitsdarlehen über 30 Jahre mit variabler Verzinsung aufnehmen, dann entspräche die Effektivverzinsung dem arithmetischen Mittelwert aller im Zeitraum von Januar 1960 bis Dezember 1989 beobachtbaren 3-Monats-Geldmarktsätzen. Bei einem Fälligkeitsdarlehen im gleichen Zeitraum mit fester Verzinsung würde der Kreditnehmer dreimal den Kreditbetrag über jeweils 10 Jahre aufnehmen. Hier berechnet sich die Effektivverzinsung als Mittelwert aus der Rendite 10-jähriger Bundesanleihen im Januar 1960, Januar 1970 und Januar 1980, d. h. man legt für jeweils 10 Jahre immer den gleichen Zins zugrunde. Zusammengefasst berechnen wir die unterschiedlichen Effektivverzinsungen für Fälligkeitsdarlehen also wie folgt (der Index $t$ bezieht sich auf Monate):

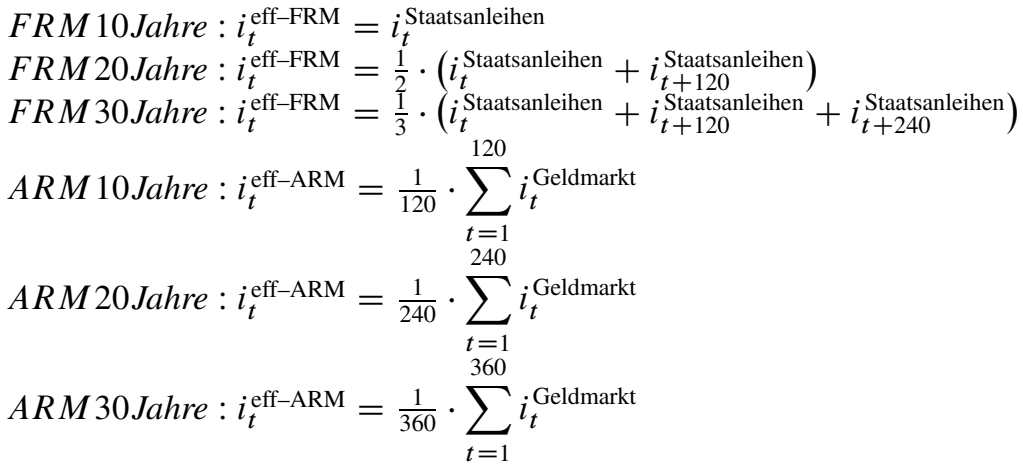

In Abb. 5 ist die effektive Verzinsung im Fall von Fälligkeitsdarlehen bei variablem Zins derjenigen mit Zinsbindung am Beispiel einer 30-jährigen Rückzahlungsdauer gegenübergestellt. Auf der Abszisse ist der Zeitpunkt des Beginns der Kreditlaufzeit dargestellt. Da der Datensatz Werte bis August 2018 enthält, endet die Zeitachse bei 30-jährigen Finanzierungszeiträumen entsprechend im September $1988 .{ }^{19}$

In der Abbildung tritt deutlich zu Tage, dass die 10-jährige Zinsbindung historisch im Durchschnitt teurer ist als eine Finanzierung mit variablen Zinsen. Vor dem Hintergrund einer über den Untersuchungszeitraum dominierenden normalen Zinsstruktur und der Tatsache, dass die generelle Zinssenkungsphase seit Anfang der 80er Jahre deutlich ausgeprägter war als die zuvor festzustellenden Zinserhö-

19 Analoge Schlussfolgerungen und qualitativen Ergebnisse würde man erhalten bei Berechnung geometrischer Durchschnitte, d.h. inkl. Zinseszinsen. 


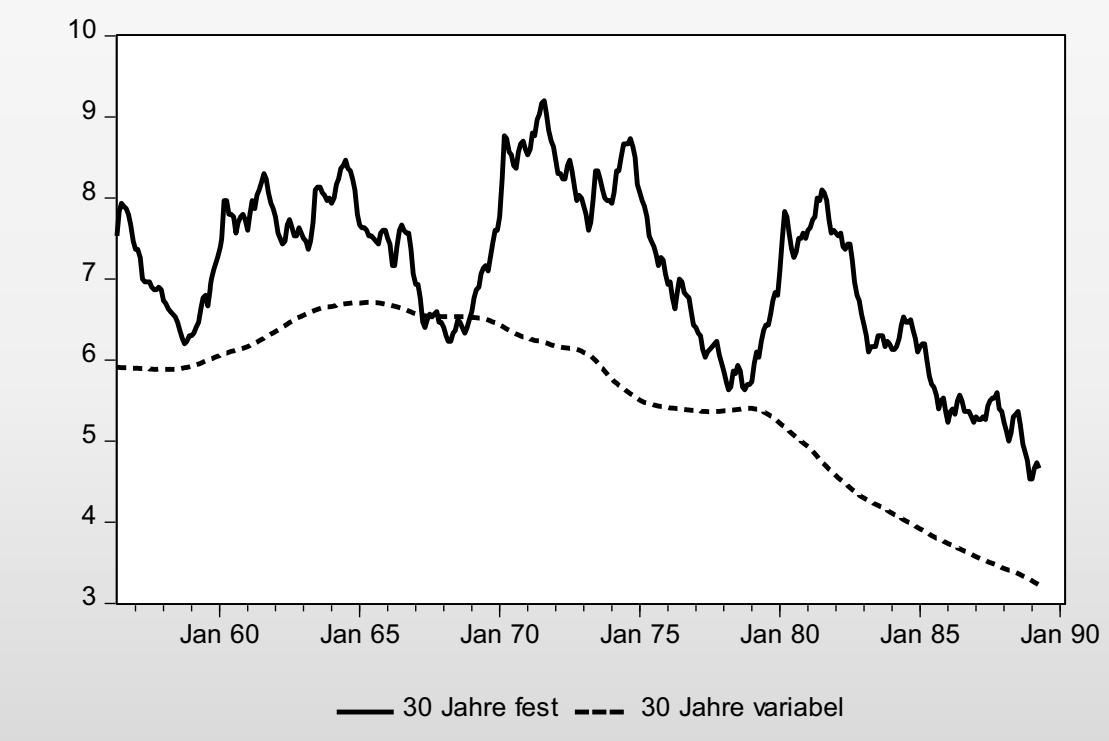

Abb. 5 Effektivverzinsung eines Fälligkeitsdarlehens bei einem Finanzierungszeitraum von 30 Jahren. Quelle: eigene Darstellung

hungen, kommt dieses Ergebnis nicht überraschend. Allerdings ist schon erstaunlich, wie häufig die variable Verzinsung besser war (siehe zudem Tab. 1). Das gilt auch für die anderen Finanzierungszeiträume. Es ist offensichtlich, dass der Zinsunterschied in Zinssenkungsphasen tendenziell höher ist als in Zinssteigerungsphasen, abhängig von der Dauer der entsprechenden Zeiträume. Aber auch in Zinssteigerungsphasen ist die Finanzierung mit variablen Zinsen im Durchschnitt günstiger. Darüber hinaus unterliegt die Finanzierung mit variablen Zinsen einer geringeren Volatilität, da in diesem Fall der glättende Effekt der Durchschnittswertberechnung durchschlägt, d.h. die Effektivverzinsung entspricht einem Durchschnitt des Geldmarktzinses aus 360 Beobachtungspunkten. In Tab. 1 sind die Ergebnisse noch einmal komprimiert zusammengefasst:

Unabhängig von der Rückzahlungsdauer ist die variable Finanzierung in über $87 \%$ der Fälle (Monate) die günstigere Finanzierungsart gewesen. Je länger der Finanzierungszeitraum, desto vergleichsweise günstiger ist eine Finanzierung mit variablen Zinsen. Die Differenz der Effektivverzinsung beträgt etwa 1,60\%-punkte. Über einen Finanzierungszeitraum von 30 Jahren würde bei einem Kreditbetrag von 1 Mio. $€$ die aufsummierte Zinsersparnis über $450.000 €$ betragen, hätte man mit variablem Zins statt mit Zinsbindung den Kredit bedient. Wie bereits erwähnt, ist überraschenderweise - die Volatilität bzw. die Stichtagsabhängigkeit bei Finanzierung mit Zinsbindung höher. Von Jahr zu Jahr schwankt die Effektivverzinsung bei 
Tab. 1 Vergleich der Effektivverzinsung eines Fälligkeitsdarlehens bei unterschiedlichen Finanzierungszeiträumen

\begin{tabular}{llll}
\hline & \multicolumn{2}{l}{ Dauer der Finanzierung } & \\
& 10 Jahre & 20 Jahre & 30 Jahre \\
\hline $\begin{array}{l}\text { Wie häufig war die variable Verzinsung besser als eine 10jäh- } \\
\text { rige Zinsbindung? }\end{array}$ & $87,60 \%$ & $93,52 \%$ & $95,37 \%$ \\
$\begin{array}{l}\text { Differenz der effektiven Verzinsung „,Fest“ vs. „Variabel“ } \\
\text { über den Gesamtzeitraum }\end{array}$ & $1,63 \mathrm{pp}$ & $1,61 \mathrm{pp}$ & $1,54 \mathrm{pp}$ \\
$\begin{array}{l}\text { Differenz der Volatilität der effektiven Verzinsung „Fest“ vs. } \\
\text { „Variabel“ über den Gesamtzeitraum }\end{array}$ & $0,56 \mathrm{pp}$ & $0,49 \mathrm{pp}$ & $0,42 \mathrm{pp}$ \\
\hline
\end{tabular}

Quelle: eigene Darstellung.

Zinsbindung um ca. 0,4-0,5\%-punkte stärker als bei variablen Zinsen. ${ }^{20}$ Der Kredit mit Zinsbindung ist also nicht nur teurer, sondern auch abhängiger vom gewählten Finanzierungszeitpunkt. ${ }^{21}$

Das Hauptargument, welches gegen eine Finanzierung mit variablen Zinsen vorgebracht wird, ist, dass diese im Vergleich zum Festzinsdarlehen unberechenbarer ist, da der Zins und damit die Zinsrate regelmäßig (in unserem Fall monatlich) angepasst werden. ${ }^{22}$ Zudem kann gerade in Zinserhöhungsphasen der variable Zins über dem langfristig gebunden Zins liegen, wodurch die finanzielle Belastung des Kreditnehmers höher ist. Liquiditätsengpässe könnten dann den Vorteil der insgesamt günstigeren kurzfristigen Finanzierung konterkarieren (siehe auch die Ausführungen im Literaturüberblick zu Beginn). Um dieses Risiko zu quantifizieren, berechnen wir die Prüfgrößen ,maximaler kumulierter Zinsgewinn“ und „maximaler kumulierter Zinsverlust“" im Fall eines Fälligkeitsdarlehens. Wir unterstellen, dass jeder Kreditnehmer mit variablem Zins ein zinsloses Konto führt, auf welches er seine monatlichen (hypothetischen) Zinsgewinne gegenüber einer Zinsbindung einzahlt bzw., falls der variable Zins über dem festen Zins liegt, dieses Konto belastet. ${ }^{23}$ Uns interessieren der größte negative Kontostand, der größte positive Kontostand und der durchschnittliche Kontostand über die gesamte Historie hinweg, die im Lauf der Finanzierung auf diesem hypothetischen Konto entstehen. Alle Werte werden als Prozentsatz der Darlehenssumme gerechnet. Der größte negative Kontostand zeigt an, welche Pufferliquidität ein Kreditnehmer (in der Vergangenheit, d.h. über den gesamten Untersuchungszeitraum) maximal hätte vorhalten müssen, um einen even-

\footnotetext{
20 Volatilität gemessen als durchschnittliche absolute jährliche Veränderung der Effektivverzinsung.

21 Diese Ergebnisse gelten qualitativ in analoger Form auch bei einem Tilgungsdarlehen, siehe Clostermann und Seitz (2018).

22 Lee (2018) spricht in diesem Zusammenhang vom „insurance benefit“, welcher bei einem Festzinsdarlehen den Nutzen des Kreditnehmers erhöht.

23 Die hypothetischen Kosten eines solchen Kontos könnte man in Anlehnung an Lee (2018) als Kosten einer „ARM insurance“ bezeichnen. Diese entsprechen dem ,insurance benefit“ eines Festzinsdarlehens. Wir unterstellen hier, dass ein Kreditnehmer bereit ist, die Zinsvorteile zu sparen. Dies widerspricht den empirischen Ergebnissen der Behavorial Finance-Literatur (siehe z. B. Thaler und Sunstein 2018, S. $148 \mathrm{ff}$.), die eine ,irrational“ hohe Gegenwartspräferenz der Individuen konstatieren. Es ist deshalb zu erwarten, dass die Zinsersparnisse bei variabler Verzinsung vermehrt wie windfall profits konsumiert werden und dadurch die Liquiditätsanspannung bei steigenden Zinsen zunimmt.
} 


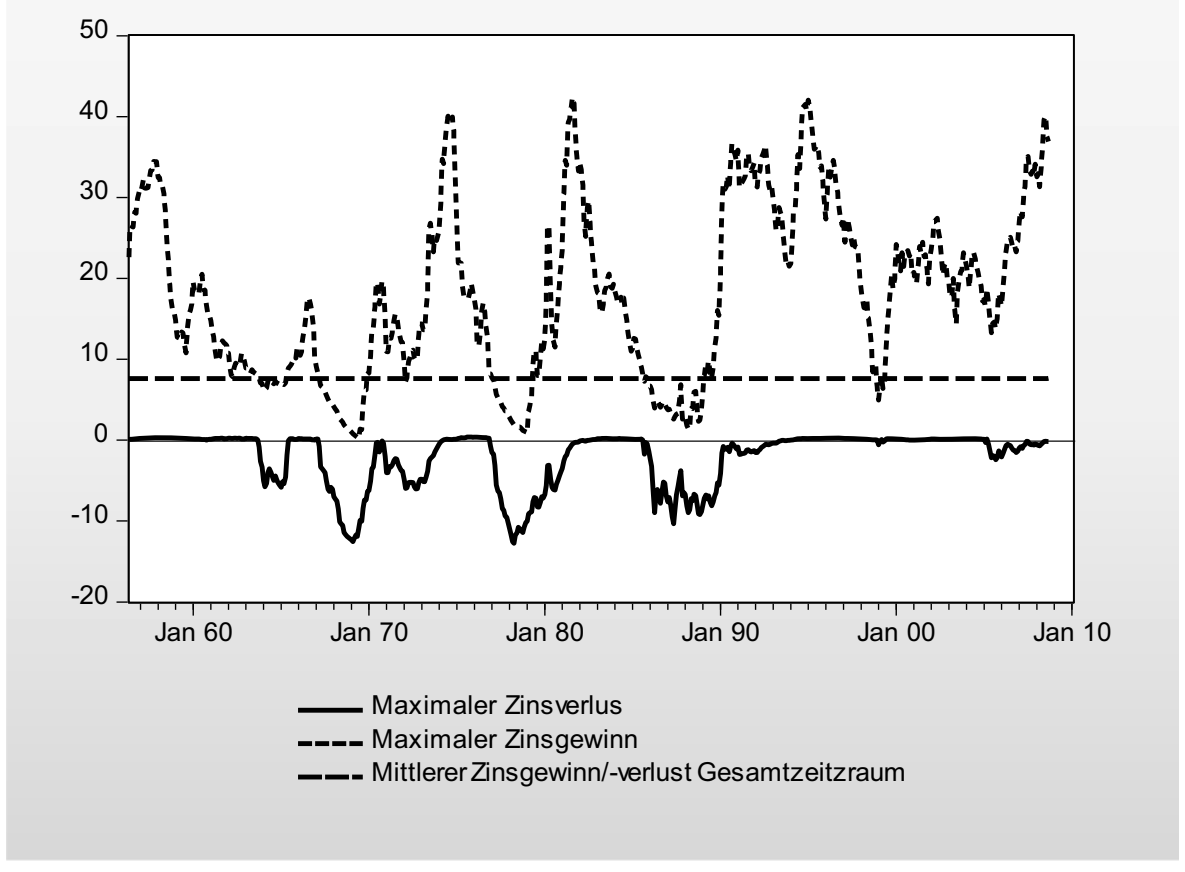

Abb. 6 Maximaler kumulierter Zinsgewinn/-verlust bei einem Finanzierungszeitraum von 10 Jahren. Quelle: eigene Darstellung

tuell temporären höheren Liquiditätsbedarf, resultierend aus einem vorübergehend im Vergleich zum Festzins höheren variablen Zins, abfangen zu können. ${ }^{24}$

In den Abb. 6, 7 und 8 ist dieser maximale kumulierte Zinsgewinn und -verlust bei unterschiedlichen Finanzierungszeiträumen dargestellt. Es fällt auf, dass sowohl der maximale kumulierte Zinsverlust als auch der maximale kumulierte Zinsgewinn und der mittlere Zinsgewinn/-verlust über dem Gesamtzeitraum mit der Länge des Finanzierungszeitraums zunimmt. Legt man einen 30-jährigen Finanzierungszeitraum zugrunde, ist der höchste historische Wert des kumulierten Zinsgewinns rund $+100 \%$, der des kumulierten Zinsverlustes rund $-20 \%$, d. h. das „Pufferkonto“ hatte in diesem Fall einen temporär maximalen negativen Kontostand von ca. $20 \%$ des Darlehensbetrags. Historisch betrachtet hätte der Kreditnehmer bei einem Fälligkeitsdarlehen mit variablem Zins im ,schlimmsten“ Fall 20\% seiner Kreditsumme ,parken“ müssen, um keine Liquiditätsengpässe zu erleiden. Die Kosten der „Puffer-Liquidität“ sind im Verhältnis zu den im Durchschnitt niedrigeren Effektivzinsen

\footnotetext{
24 Diese Überlegungen wären durch die Einkommensentwicklung zu ergänzen, da durch Lohnsteigerungen eine deutliche Liquiditätsentspannung entstehen kann. Das „,wahre“ Liquiditätsrisiko wird deshalb bei unseren Berechnungen überschätzt. Gemessen an den Stundenlöhnen im Verarbeitenden Gewerbe in Deutschland betrugen die durchschnittlichen jährlichen Lohnsteigerungen in den sechs Dekaden seit 1960 $7,1 \%, 7,3 \%, 3,6 \%, 3,3 \%, 1,6 \%$ und 2,3\% (qualitativ ähnlich würde die Situation bei Orientierung am Arbeitnehmerentgelt je Arbeitnehmer in der Gesamtwirtschaft aussehen). Gerade in den Hochzinsphasen hatte man also auch relativ hohe Einkommenszuwächse.
} 


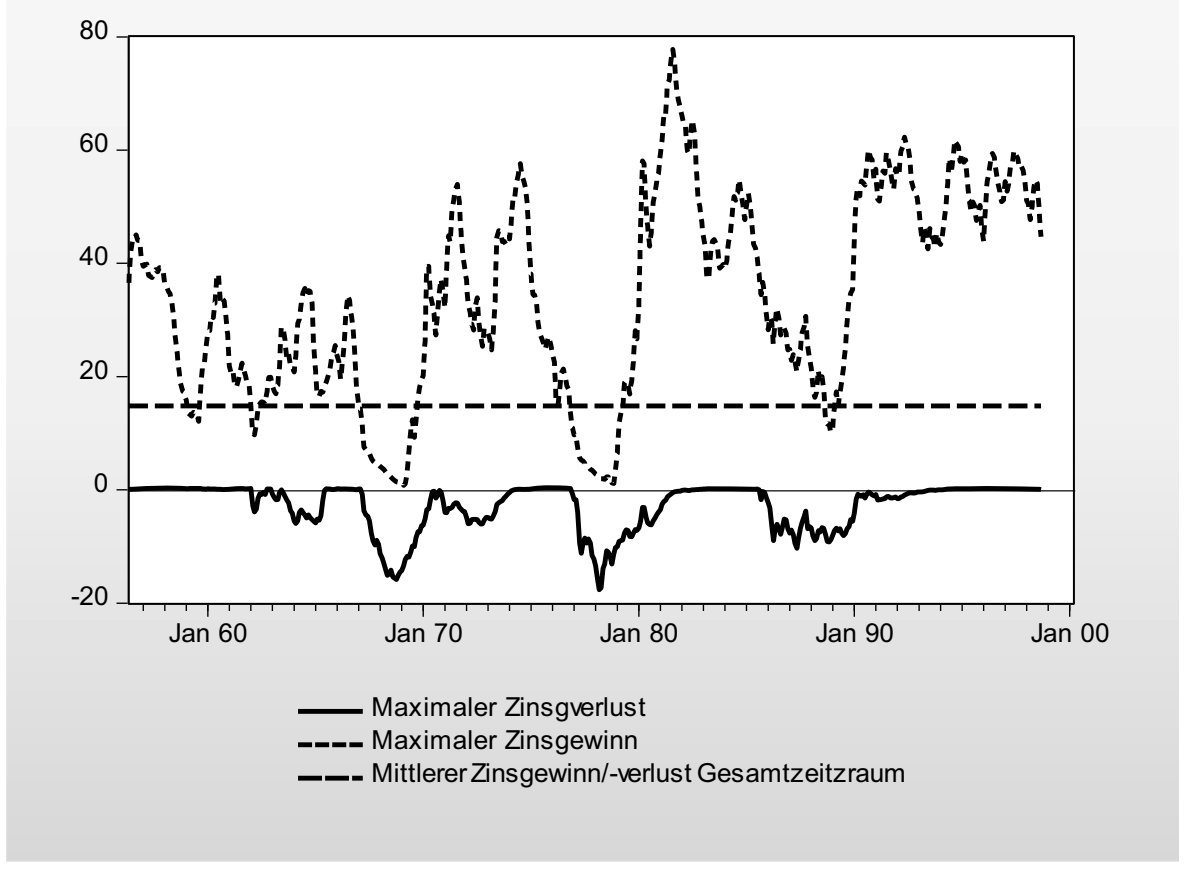

Abb. 7 Maximaler kumulierter Zinsgewinn/-verlust bei einem Finanzierungszeitraum von 20 Jahren. Quelle: eigene Darstellung

bei Finanzierung mit variablem Zins allerdings ,verschmerzbar“. Unterstellt man, dass der Schuldner eine um 20\% höhere Kreditsumme aufnimmt und diese $20 \%$ auf einem zinslosen Girokonto ,parkt“, dann erhöht sich die Effektivverzinsung seines Kredits durchschnittlich über den gesamten Datenzeitraum gerechnet um 1,1\%punkte. Vergegenwärtigt man sich, dass ein Darlehen mit variabler Verzinsung um über 1,5\%-punkte günstiger als ein Kredit mit Festzinsvereinbarung ist, halten sich die Kosten der „Puffer-Liquidität“ im Rahmen, zumal das gewählte Beispiel eine historische Extremsituation ist und die Annahme, dass die Pufferliquidität zinslos gehalten wird, ein ,worst case“-Szenario darstellt. In über $90 \%$ der Fälle liegt der maximale Zinsverlust bei weniger als $10 \%$ der Darlehenssumme. Darüber hinaus beträgt der mittlere Zinsgewinn/-verlust bei einem Finanzierungszeitraum von 30 Jahren etwa $+20 \%$, d. h. durchschnittlich erzeugt die variable Verzinsung keinen Liquiditätsbedarf, sondern im Gegenteil sogar Liquiditätsüberschüsse von durchschnittlich $20 \%$ des Darlehensbetrags. ${ }^{25}$ In Summe bleibt also die Schlussfolgerung, dass historisch betrachtet in Deutschland in dem hier zugrunde gelegten Zeitraum Darlehen mit variablen Zinssätzen für den Kreditnehmer günstiger waren, erhalten.

25 Darüber hinaus zeigen gesonderte Berechnungen, dass der Endkontostand des Pufferkontos im Durchschnitt ca. $50 \%$ betrug. D. h. im Mittel müssen Kreditnehmer mit variabler Verzinsung $50 \%$ weniger tilgen. 


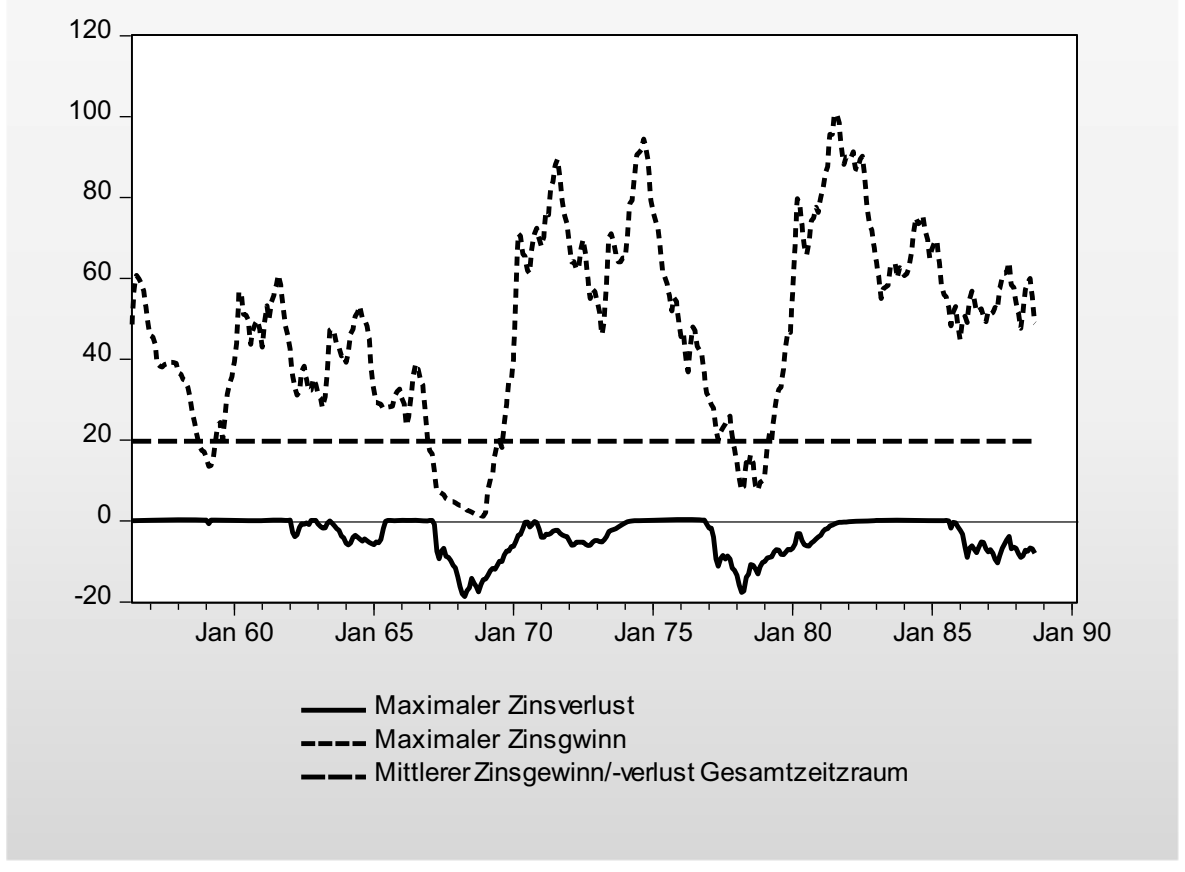

Abb. 8 Maximaler kumulierter Zinsgewinn/-verlust bei einem Finanzierungszeitraum von 30 Jahren. Quelle: eigene Darstellung

\section{Zusammenfassung, Schlussfolgerungen}

Gegenstand der vorliegenden Untersuchung war es, zu beurteilen, ob Kredite mit Zinsbindung oder Kredite mit variabler Verzinsung für Kreditnehmer in Deutschland über einen Zeitraum von 60 Jahren historisch betrachtet günstiger oder teurer waren. Als Referenzzinssatz (orientiert an der Schweizer Situation) für den Zins für Kredite mit Zinsbindung wählten wir den Zins für 10-jährige Staatsanleihen, für die variable Verzinsung den 3-Monats-Geldmarktsatz. Bei den Berechnungen differenzierten wir zwischen 10-, 20- und 30-jährigen Finanzierungszeiträumen. Während bei der Finanzierung mit variablen Zinsen der Zins (annahmegemäß) monatlich angepasst wird, unterstellten wir bei der Finanzierung mit fixen Zinsen eine maximale Zinsbindung von 10 Jahren. Es zeigte sich, dass ein Kredit mit permanent variabler Verzinsung eine niedrigere Effektivverzinsung als ein Kredit mit fester Zinsbindung hat. Je länger der zugrunde gelegte Finanzierungszeitraum ist, desto deutlicher zeigt sich aus Sicht des Kreditnehmers die Überlegenheit der variablen Verzinsung. Darüber hinaus war auch die Volatilität der Effektivverzinsung in diesem Fall geringer. Eine Finanzierung mit variablem Zins war weniger abhängig vom gewählten Startzeitpunkt. Um dem Argument Rechnung zu tragen, dass die Zinsbelastung bei einer variablen Finanzierung temporär höher sein könnte, haben wir in der Analyse die Kosten einer sogenannten „Puffer-Liquidität“ berücksichtigt. Allerdings ändern sich dadurch die generellen Schlussfolgerungen nicht. 
In den letzten Jahren ist die Zinsstrukturkurve nicht zuletzt durch die expansive Geldpolitik der Zentralbanken (Nullzinspolitik, Quantitative Easing) flacher geworden ist (siehe z.B. Cœuré 2017). Vor diesem Hintergrund könnte eine zukünftige Stoßrichtung für weitere Analysen diesem Aspekt verstärkt Rechnung tragen. Tatsächlich würde eine flachere Zinsstrukturkurve den Vorteil einer Finanzierung mit variablen Zinssätzen vermindern. Gleichzeitig scheint die Volatilität der Zinsstruktur geringer geworden sein. So betrug die Standardabweichung des Spreads über den gesamten hier betrachteten Beobachtungszeitraum 1,3\%-punkte, in den 1960er und 1970ern 1,65\%-punkte, während sie seit 2010 nur noch gut einen \%-punkt ausmachte. Das daraus resultierende geringere Zinsänderungsrisiko würde die Vorteilhaftigkeit einer Finanzierung mit variablen Zinssätzen erhöhen. Es bleibt also letztendlich die Frage zu beantworten, wie das zukünftige Zinsszenario aussehen könnte bzw. was die neue Zinsnormalität darstellt. Bleiben die Zinsen auf längere Zeit auf dem aktuell extrem niedrigen Niveau? Kommen wir zu den Zinsniveaus zurück, wie sie vor der Finanzkrise vorherrschten? Wie volatil werden die Zinsen zukünftig sein? Und was bedeutet dies konkret für die Entscheidung fixe versus variable Finanzierung?

Schließlich haben wir uns auch nicht mit Finanzierungsstrategien auseinandergesetzt, die beide Finanzierungsarten - variable Zinsen und Zinsbindung - geschickt kombinieren, um einen überlegeneren Mix aus Effektivverzinsung und Volatilität zu finden. Auch die Möglichkeit mit Hilfe von Zinsabsicherungsinstrumenten die Finanzierung zu optimieren, bleibt weiterer Forschung vorbehalten.

Open Access Dieser Artikel wird unter der Creative Commons Namensnennung 4.0 International Lizenz (http://creativecommons.org/licenses/by/4.0/deed.de) veröffentlicht, welche die Nutzung, Vervielfältigung, Bearbeitung, Verbreitung und Wiedergabe in jeglichem Medium und Format erlaubt, sofern Sie den/die ursprünglichen Autor(en) und die Quelle ordnungsgemäß nennen, einen Link zur Creative Commons Lizenz beifügen und angeben, ob Änderungen vorgenommen wurden.

\section{Literatur}

Andrews D, Caldera Sánchez A (2011) The evolution of homeownership rates in selected OECD countries: demographic and public policy influences. J Econ Stud 2011/1:207-243

Andrews D, Caldera Sánchez A, Johansson Å (2011) Housing markets and structural policies in OECD countries. OECD economics department working papers no. 836. OECD Publishing, Paris

Badarinza C, Campbell J, Ramadorai T (2017) What calls to ARms? International evidence on interest rates and the choice of adjustable-rate mortgages. Manage Sci. https://doi.org/10.1287/mnsc.2016. 2629

Badarinza C, Campbell JY, Ramadorai T (2016) International comparative household finance. Annu Rev Econ 8:111-144

Basten C, Guin B, Koch C (2017) How do banks and households manage interest rate risk? Evidence from the Swiss mortgage market. CESifo Working Paper 6649

Calza A, Monacelli T, Stracca L (2012) Housing finance and monetary policy. J Eur Econ Assoc 11:101-122

Campbell J, Cocco J (2003) Household risk management and optimal mortgage choice. Q J Econ 118:1449-1494

Campbell J, Cocco J (2015) A model of mortgage default. J Finance 70:1495-1554

Clostermann J, Seitz F (2018) Feste Zinsbindung versus kurzfristig variable Zinskonditionen in Deutschland. Die Hochschule im Dialog: Weidener Diskussionspapiere Nr. 62

Cœuré B (2017) Dissecting the Yield Curve: a central bank perspective. Frankfurt, 16. Mai 2017. welcome remarks at the annual meeting of the ECB's Bond Market Contact Group 
Deutsche Bank Research (2019) Häuslebauer finanzieren immer langfristiger (Deutschland Monitor, Finanzen der privaten Haushalte, 12. Juni 2019)

Deutsche Bundesbank (2002) Zum Zusammenhang zwischen Kreditzinsen deutscher Banken und Markzinsen, Monatsbericht März, S 53-66

Deutsche Bundesbank (2019a) Monatsbericht, Statistischer Teil: IV. Banken, Mai

Deutsche Bundesbank (2019b) Zinsstatistik

Ehrmann M, Ziegelmeyer M (2017) Mortgage choice in the Euro area: macroeconomic determinants and the effect of monetary policy on debt burdens. J Money Credit Bank 49:469-494

Europäische Zentralbank (2007) Änderungen der Bankkreditzinsen und Wohnungsbaukredite (Neugeschäft) im Euro-Währungsgebiet, Kasten 3, Monatsbericht März, 46-48

Foà G, Gambacorta L, Guiso L, Mistrulli PE (2015) The supply side of housing finance. BIS working papers no 531

IWF (2004) World economic outlook: the global demographic transition

Koijen R, van Hemert O, van Nieuwerburgh S (2009) Mortgage timing. J financ econ 93:292-324

Lee K (2018) Fixed-rate mortgages, labor markets, and efficiency. J Money Credit Bank 50:1033-1072

Lerbs O, Voigtländer M (2018) Ist eine makroprudenzielle Regulierung des deutschen Hypothekenmarktes geboten? Perspekt Wirtschaftspolit 19(1):42-56

Moench E, Vickery J, Aragon D (2010) Why is the market share of adjustable-rate mortgages so low? Fed Reserv Bank N Y Curr Issues Econ Finance 16:1-11

Mugerman Y, Ofir M, Wiener Z (2016) How homeowners choose between fixed and adjustable rate mortgages? Quart J of Fin 6:1-21

Paiella M, Pozzolo AF (2007) Choosing between fixed and adjustable rate mortgage. In: Agarwal S, Ambrose BW (Hrsg) Household credit usage: personal debt and mortgages. Palgrave, New York, S 219-236

Rachel L, Smith TD (2017) Are low real interest rates here to stay? Int J Cent Bank 13(3):1-42

Rampini A, Viswanathan S (2016) Household risk management. NBER Working Paper 22293.

Rubio M (2011) Fixed- and variable-rate mortgages, business cycles, and monetary policy. J Money Credit Bank 43:657-688

SNB (2019) Zinssätze und Devisenkurse, Publizierte Zinssätze für Neugeschäfte

Thaler RH, Sunstein CR (2018) Nudge - Wie man kluge Entscheidungen anstößt, 13. Aufl. Ullstein, Berlin 